Palavras chave:

Modelos explícitos ou globais

Desbaste

Idade técnica de corte

Índice de sítio

Histórico:

Recebido 15/03/2012

Aceito $21 / 01 / 2015$

Keywords:

Explicit system of production

Thinning

Technical cutting age

Site index

Correspondência: andias@irati.unicentro.br
Flávio Augusto Ferreira do Nascimento', Andrea Nogueira Dias², Afonso Figueiredo Filho $^{2}$, Gabriel de Magalhães Miranda², Julio Eduardo Arce'

\section{SISTEMA DE CRESCIMENTO E PRODUÇÃO PARA POVOAMENTOS DE Pinus taeda NA REGIÃO NORTE DE SANTA CATARINA}

RESUMO: Objetivou-se, neste trabalho, testar diferentes modelos matemáticos para o ajuste de curvas de índice de sítio e avaliar o modelo de Clutter para predição do crescimento e da produção em povoamentos de Pinus taeda na região norte do Estado de Santa Catarina. Os dados de altura dominante foram obtidos por meio da medição de 216 parcelas, com área de $400 \mathrm{~m}^{2}$, em 2009. Para o modelo de Clutter, utilizaram-se dados de inventário contínuo, realizado entre os anos de 2004 e 2008, em talhões com idades entre 5 e 34 anos. As estatísticas coeficiente de determinação ajustado $\left(R^{2}{ }_{j j}\right)$, erro padrão da estimativa $\left(S_{y x}\right)$, erro padrão da estimativa em porcentagem $\left(S_{y x} \%\right)$ e distribuição de resíduos em porcentagem foram utilizadas para selecionar o melhor modelo. Verificouse que o modelo de sítio mais adequado foi o de Silva-Bailey e o modelo de Clutter foi considerado eficiente para estimar o crescimento e a produção $\mathrm{em} \mathrm{m}^{3} \cdot \mathrm{ha}^{-1}$, do ponto de vista estatístico e biológico.

\section{A SYSTEM FOR Pinus taeda GROWTH AND YIELD IN THE NORTH OF SANTA CATARINA}

ABSTRACT: The objective of this study was to test different models for fitting site index curves and evaluat the Clutter model for predicting Pinus taeda growth and yield in the northern State of Santa Catarina. The dominant height data were obtained from 216 plots measured in 2009, with an area of $400 \mathrm{~m}^{2}$. To fit the Clutter model we used data from permanent plots, conducted between 2004 and 2008 in areas aged between 5 and 34 years. The coefficient of determination $\left(R^{2}{ }_{a d j}\right)$, standard error of estimate $\left(S_{y x}\right)$, standard error of estimate in percentage $\left(S_{y x} \%\right)$ and residual percentage distribution were the statistics used to select the best model. The Silva-Bailey model was the most appropriated to represent site index curves, while the Clutter model performed well for estimating the growth and yield $\left(\mathrm{m}^{3} \cdot \mathrm{ha}^{-1}\right)$, from a statistical and biological point of view. 


\section{INTRODUÇÃO}

As decisões na área florestal abrangem diversas situações onde é essencial ter informações referentes ao estoque futuro dos povoamentos. Tais casos ocorrem na definição do momento ideal para o desbaste e o corte raso, na compra e venda da madeira, na aquisição de novas áreas, entre outras. Nesse sentido, a modelagem do crescimento e da produção, aliada a dados econômicos são elementos fundamentais para o planejamento florestal.

Clutter et al. (1983) classificaram os modelos de crescimento e produção, que fornecem estimativa do volume por unidade de área, como sendo do tipo sistema de produção explícito. Segundo Vanclay (1994), estes são modelos de crescimento e produção, nos quais a unidade básica de modelagem são parâmetros do povoamento, como a área basal, alguma medida do grau de utilização da área por árvores, volume e parâmetros caracterizando a distribuição diamétrica.

De acordo com Campos e Leite (2009), o modelo de crescimento e produção desse tipo mais difundido no Brasil é o modelo de Clutter. Aplicações desse modelo são encontradas para povoamentos de eucalipto não desbastados, como, por exemplo, os trabalhos de Cruz et al. (2008), Resende et al. (2004) e Santana et al. (2005).

O modelo de Clutter também foi aplicado em povoamentos de eucalipto com desbaste, podendo-se citar, nesse caso, os trabalhos de Dias (2000) e Dias et al. (2005a, 2005b). Apesar de aplicado a povoamentos desbastados, ○ modelo de Clutter não diferencia tendências de crescimento pós-desbaste. Estudos foram conduzidos visando a avaliar essa característica do modelo. Dias (2005) avaliou a eficiência do modelo para descrever tendências de crescimento e produção pósdesbaste em povoamentos de eucalipto. Gorgens et al. (2007) concluíram que o modelo de Clutter, em sua forma original, pode ser usado sem prejuízo para a simulação de desbastes, mesmo não diferenciando tendências de crescimento.

Objetivou-se, neste trabalho testar diferentes modelos matemáticos para ajuste de curvas de índice de sítio e modelar um sistema de crescimento e produção para povoamentos de Pinus taeda na região norte do Estado de Santa Catarina.

\section{MATERIAL E MÉTODOS}

\section{Fonte de dados}

A pesquisa foi realizada com dados provenientes de povoamentos de Pinus taeda, pertencentes ao banco de dados de uma empresa florestal, localizada na região norte do estado de Santa Catarina. O clima predominante na região, segundo a classificação de Köeppen, é do tipo $\mathrm{Cfb}$. O espaçamento de plantio empregado pela empresa é de 2,5 × 2,5 m (1600 árvores'ha $\left.{ }^{-1}\right)$. Quanto ao manejo, a empresa tem registrado em seu histórico o teste de diferentes configurações para os desbastes e cortes rasos. No entanto, para os plantios realizados a partir de 1998 tem-se buscado realizar o manejo com um desbaste aos 10 aos de idade e corte raso aos 17 anos. Nos desbastes realizados, até então, retiraram-se entre 30 a 50\% da área basal. Na Tabela I, são apresentados os valores médios de área basal, volume e número de árvores por hectare em função da idade da floresta em estudo. A instalação de parcelas para medição da altura dominante $(\mathrm{Hdom})$ foi necessária em função do inventário contínuo realizado pela empresa não levantar essa variável.

TABELA 1 Dados da floresta em estudo e relação de parcelas utilizadas.

TABLE 1 Data form the forest and plots used in the study.

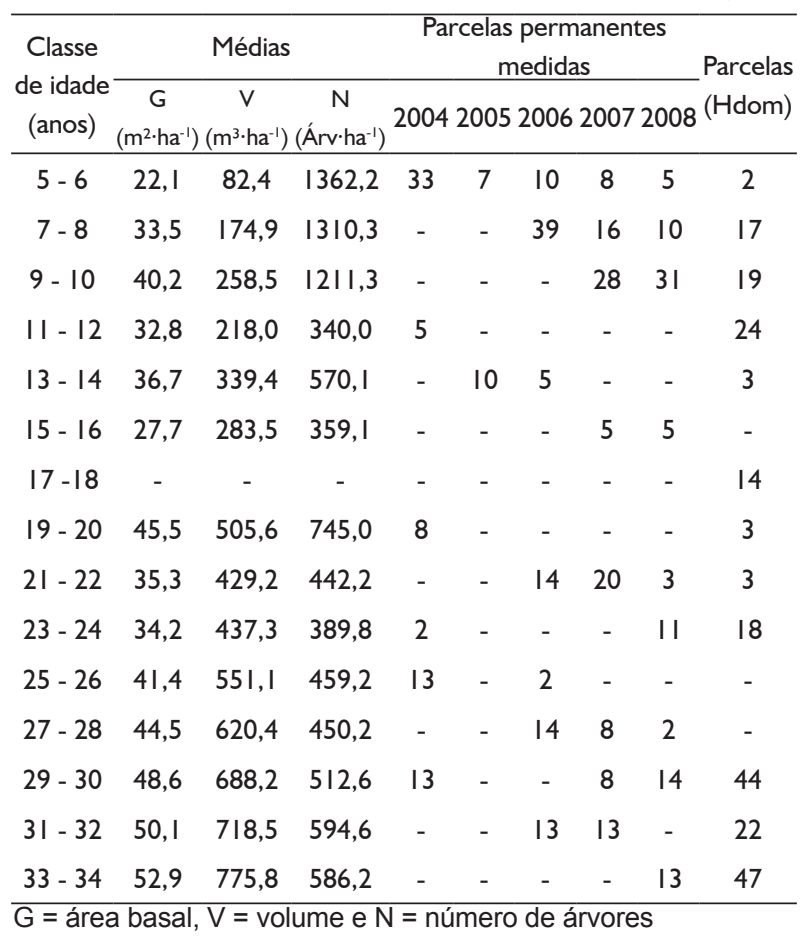

\section{Modelos de índice de sítio}

Para fazer o ajuste dos modelos de sítio, mediram-se as alturas dominantes de 216 parcelas, entre os meses de janeiro e junho de 2009. Em cada parcela de $20 \times 20$ metros $\left(400 \mathrm{~m}^{2}\right)$ foram medidas as alturas de quatro árvores dominantes, sendo a média utilizada no ajuste dos modelos. Por serem informações de um único 
ano, considerou-se que os dados foram provenientes de parcelas temporárias para efeito de ajuste dos modelos de sítio.

Foram testados seis modelos de sítio e as curvas foram construídas pelo método da curva-guia. Na Tabela 2 , são apresentados os modelos e seus respectivos autores, conforme pesquisa de Martins et al. (2007). Todos os ajustes foram realizados por meio de regressão não-linear, exceto o modelo de Schumacher, o qual foi ajustado com regressão linear. $\mathrm{Na}$ Tabela $2 \mathrm{Hdom}=$ altura média das quatro árvores dominantes da parcela, em $\mathrm{m} ; \mathrm{I}=$ idade do povoamento, em anos; $I_{i}=$ idade índice, em anos; e $S=$ índice de sítio.

\section{Modelo de Crescimento e Produção}

A base de dados utilizada para o ajuste do modelo de Clutter (CLUTTER, 1963) refere-se a remedições realizadas em 216 parcelas permanentes nos anos de 2004, 2006, 2007 e 2008, contemplando talhões com idades entre 5 a 33 anos. Em cada medição foi registrado o diâmetro à altura do peito (DAP) de todas as árvores contidas nas parcelas. $\mathrm{O}$ ajuste dos modelos I e 2 foi realizado pelo procedimento estatístico denominado Mínimos Quadrados em Dois Estágios, onde: $V_{2}=$ volume, em $\mathrm{m}^{3} \cdot \mathrm{ha}^{-1}$, na idade $I_{2} ; A B_{1}=$ área basal, em $\mathrm{m}^{2} \cdot \mathrm{ha}^{-1}$, na idade $I_{1} ; \quad A B_{2}=$ área basal, em $\mathrm{m}^{2} \cdot \mathrm{ha}^{-1}$, na idade $I_{2} ; I_{1}=$ Idade atual do povoamento em anos; $I_{2}=$ Idade futura do povoamento em anos; $S=$ índice de sítio, em metros; $a$ e $b=$ parâmetros a estimar.

$$
\begin{aligned}
& \ln \left(V_{2}\right)=\beta_{0}+\frac{\beta_{1}}{I_{2}}+\beta_{2} \cdot S+\beta_{3} \cdot \ln \left(A B_{2}\right) \\
& \ln \left(A B_{2}\right)=\ln \left(A B_{1}\right) \cdot\left(\frac{I_{1}}{I_{2}}\right)+\alpha_{0} \cdot\left(1-\frac{I_{1}}{I_{2}}\right)+\alpha_{1} \cdot\left(1-\frac{I_{1}}{I_{2}}\right) \cdot S
\end{aligned}
$$

\section{Avaliação dos modelos}

A avaliação dos modelos foi realizada com base no Coeficiente de Determinação ajustado $\left(R_{a j}^{2}\right)$, Erro Padrão de Estimativa absoluto $\left(S_{y x}\right)$ e relativo $\left(S_{y x} \%\right)$. No caso do modelo de Clutter, por se tratar de modelos logarítmicos, as estatísticas de $S_{y x}$ e $S_{y x} \%$ foram

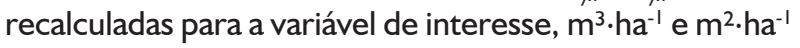
respectivamente, para o modelo de predição do volume futuro e área basal futura, conforme (3) e (4), em que: $\bar{Y}$ $=$ média dos valores observados; $Y_{i}=$ variável observada; $\hat{Y}_{i}=$ variável estimada; $n=$ número de observações; e $p$ = número de coeficientes do modelo.

$$
\begin{aligned}
& S_{y x}=\sqrt{\sum_{i=1}^{n}\left(Y_{i}-\hat{Y}_{i}\right) /(n-p)} \\
& S_{y x} \%=S_{y x} / \bar{Y} .100
\end{aligned}
$$

Também foi realizada a análise de resíduos, sendo que os desvios ou resíduos foram calculados por meio da equação 5 , em que: $\operatorname{Res}(\%)=$ resíduo em

\begin{tabular}{|c|c|c|}
\hline Autor & Modelo original & Modelo guia \\
\hline Schumacher & Hdom $=\beta_{0} \cdot \exp \left(\frac{\beta_{1}}{\mathrm{I}}\right)$ & Hdom $=S \cdot \exp \left[\beta 1 \cdot\left(\frac{1}{\mathrm{I}}-\frac{1}{\mathrm{l}_{\mathrm{i}}}\right)\right]$ \\
\hline Chapman-Richards & Hdom $=\beta_{0} \cdot\left[1-\exp \left(-\beta_{1} \cdot \mathrm{I}\right)\right]^{\beta_{2}}$ & Hdom $=S \cdot\left[\frac{1-\exp \left(-\beta_{1}{ }^{\prime}\right)}{1-\exp \left(-\beta_{1}{ }^{\mathrm{li}}\right)}\right]^{\beta_{2}}$ \\
\hline Silva-Bailey & Hdom $=\beta_{0} \cdot \exp \left(\beta_{1} \cdot \beta_{2}^{l}\right)$ & Hdom $=S \cdot \exp \left[\beta_{1} \cdot\left(\beta_{2}^{l}-\beta_{2}^{l i}\right)\right]$ \\
\hline Mitscherlich & Hdom $=\beta_{0}-\beta_{1} \cdot \beta_{2} \mathrm{I}$ & Hdom $=S-\beta_{1} \cdot\left(\beta_{2}{ }^{l}-\beta_{2}{ }^{l i}\right)$ \\
\hline Weibull & Hdom $=\beta_{0}-\beta_{1} \cdot \exp \left(\beta_{2} . \beta_{3}\right)$ & Hdom $=S-\left[\beta_{1} \cdot \exp \left(-\beta_{2} \cdot \mid \beta_{3}\right)-\beta_{1} \cdot \exp \left(-\beta_{2} \cdot \mathrm{li}_{3} \beta_{3}\right)\right]$ \\
\hline Clutter-Jones & Hdom $=\beta_{0} \cdot\left(1+\beta_{1} \cdot \beta_{2}\right)^{\beta_{3}}$ & Hdom $=S \cdot\left(\frac{1+\beta_{1} \cdot \mid \beta_{2}}{1+\beta_{1} \cdot \mathrm{I}_{\mathrm{i}} \beta_{2}}\right)^{\frac{1}{\beta_{3}}}$ \\
\hline
\end{tabular}
percentagem; $Y_{i}$ e $\hat{Y}_{i}$ explicadas, anteriormente.

TABELA 2 Modelos testados para expressar o índice de sítio.

TABLE 2 Models tested to represent the site index 
$\operatorname{Res}(\%)=\left(\frac{Y_{i}-\hat{Y}_{l}}{Y_{i}}\right) \cdot 100$

O modelo de Clutter também foi avaliado com base nos fundamentos biológicos, ou seja, o que se espera é que quanto melhor o sítio mais cedo ocorra a idade técnica de corte (ITC), a qual é representada pelo ponto de cruzamento entre as curvas de incremento corrente anual (ICA) e incremento médio anual (IMA). Assim, as curvas de IMA e ICA foram construídas para diferentes índices de sítio, a fim de verificar seus efeitos sobre a ITC.

\section{Curvas de produção}

Para a construção das curvas de produção, - modelo de predição da área basal futura requer um valor de área basal inicial. Para tanto, utilizou-se uma metodologia similar àquela empregada por Dias et al. (2005a). Na presente pesquisa, considerou-se a área basal inicial média aos 5 anos para o sítio 23, área basal inicial média menos um desvio padrão para o sítio 20 e área basal inicial média mais um desvio padrão para o sítio 26.

\section{RESULTADOS E DISCUSSÃO}

\section{Ajuste dos Modelos de Sítio}

$\mathrm{Na}$ Tabela 3, são apresentadas as estatísticas e coeficientes de ajuste dos modelos de sítio.

Como forma de escolher o modelo mais adequado para estimar o índice de sítio, construiu-se a distribuição gráfica dos resíduos dos dois melhores

TABELA 3 Coeficientes e estatísticas dos ajustes dos modelos de sítio.

TABLE 3 Coefficients and statistics of the fitted site models.

\begin{tabular}{|c|c|c|c|c|}
\hline Autor & Modelo & $\mathrm{R}_{\text {aj }}^{2}$ & $\mathrm{~S}_{\mathrm{yx}}(\mathrm{m})$ & $\mathrm{S}_{\mathrm{yx}}(\%)$ \\
\hline Silva-Bailey & $\begin{array}{l}\text { Hdom }=33,7963 . \\
\exp \left(-2,6076 \cdot 0,8957^{\prime}\right)\end{array}$ & 0,9493 & 1,9068 & 7,6382 \\
\hline $\begin{array}{l}\text { Chapman- } \\
\text { Richards }\end{array}$ & $\begin{array}{l}\text { Hdom }=34,9032 \\
{[1-\exp (-0,0848 \cdot 1)]^{1,5907}}\end{array}$ & 0,9495 & 1,9660 & 7,8752 \\
\hline Weibull & $\begin{array}{l}\text { Hdom }=8,1436+35,9599 \cdot \exp \\
\left(-27,\left.917 \cdot\right|^{-1,2}\right)\end{array}$ & 0,9485 & 1,9835 & 7,9407 \\
\hline Mitscherlich & Hdom $=36,5847-42,2245 \cdot 0,9371^{\prime}$ & 0,9471 & 2,0044 & 8,0291 \\
\hline Clutter-Jones & $\begin{array}{l}\text { Hdom }=59,2704 \\
{\left[1-1,\left.1146 \cdot\right|^{-0,5917}\right]^{4,1770}}\end{array}$ & 0,9325 & 2,0898 & 8,3192 \\
\hline Schumacher & Hdom $=3,7493 \cdot \exp (-10,3825 / I)$ & 0,9292 & 2,1216 & 8,4988 \\
\hline
\end{tabular}

modelos, respectivamente, o de Silva-Bailey (Figura Ia) e o de Chapman-Richards (Figura Ib). Constatou-se que ambos os modelos apresentaram estatísticas e resíduos similares, assim, a escolha do modelo para construir as curvas de sítio recaiu para o modelo de Silva-Bailey que apresentou erro médio mais baixo.

Os coeficientes de ajustes dos modelos apresentados na Tabela 2 estão próximos aos encontrados por Téo et al. (20l I). Esses pesquisadores verificaram que o modelo Monomolecular $\left(R^{2}{ }_{a j}=0,97 ; S_{y x}=1,8608\right)$ e o de Chapman-Richards $\left(R^{2}{ }_{a j}=0,97 ; S_{y x}=1,8615\right)$. Outras pesquisas com $P$. taeda e $P$. elliottii na região sul do Brasil verificaram melhores resultados com o modelo de Chapman-Richards (SCOLFORO; MACHADO, 1988; SELLE et al., 1994; TONINI et al., 2002).

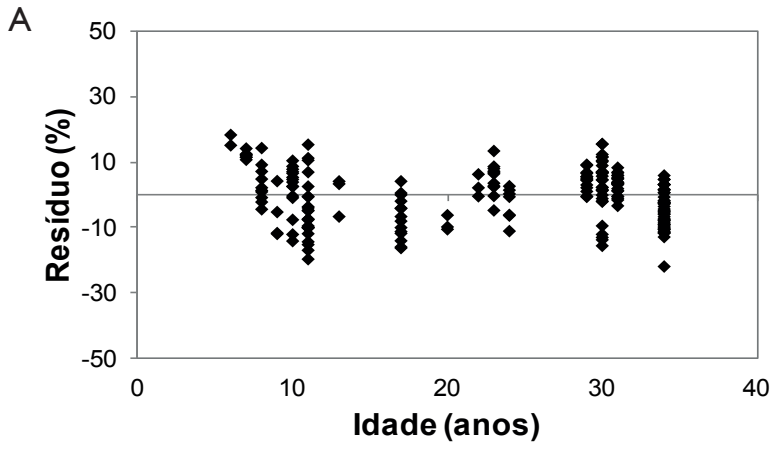

B

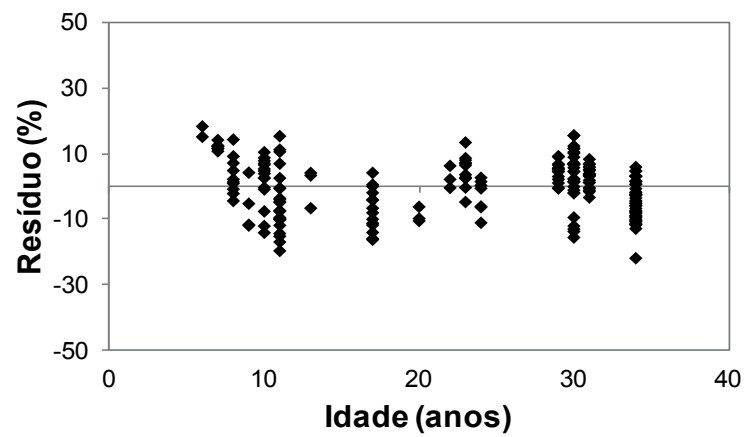

A- Modelo de Silva-Bailey; B- Modelo de Chapman-Richards

FIGURA 1 Distribuição gráfica dos resíduos para os dois melhores modelos para representar as curvas de índice de sítio.

FIGURE 1 Graphic distribution of the residues for the two best models to represent the site index curves.

\section{Construção das Curvas de Sítio}

Na Figura 2, são apresentadas as curvas de sítio construídas com o modelo selecionado. Para facilitar a visualização dos dados, as curvas foram divididas em três classes com valores centrais de sítio igual a 20, 23 e 26 considerando a idade-índice de 17 anos. 


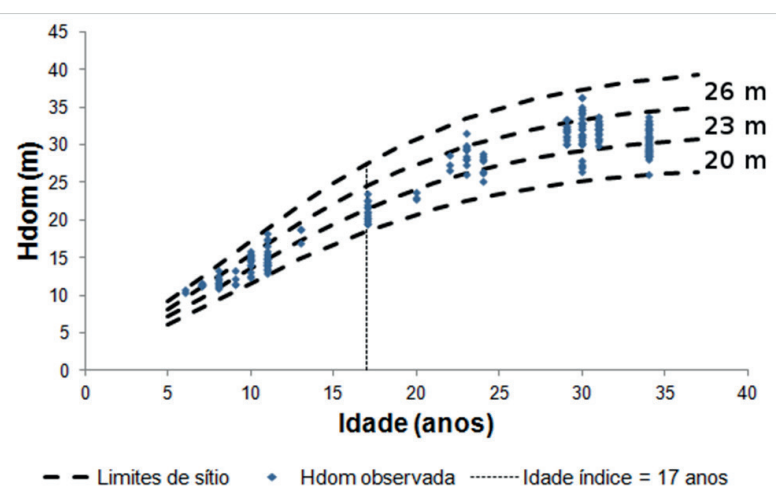

FIGURA 2 Curvas de índice de sítio construídas com o modelo de Silva-Bailey.

FIGURE 2 Site index curves built with the Silva-Bailey model.

A amostragem das alturas dominantes com parcelas temporárias, utilizada neste trabalho, pressupõe que todos os sítios estejam amostrados em todas as idades, no entanto, algumas falhas nessa amostragem podem ser observadas na Figura 2. Isso ocorreu em função da estrutura de idades da floresta, ou seja, não havia talhões com determinadas idades na época das medições. Dessa forma, essas curvas geradas não devem ser consideradas definitivas e podem ser melhoradas, intensificando-se a amostragem em diferentes ocasiões.

$\mathrm{Na}$ presente pesquisa, optou-se pela idade índice de 17 anos por esta ser a idade, atualmente, utilizada pela empresa para as rotações. Em outras pesquisas, por exemplo, tem-se as idade índice de 25 anos (SCOLFORO; MACHADO, 1988), 20 anos (SELLE et al., 1994), 18 anos (TONINI et al., 2002), 24 anos (TÉO et al., 20I I) e I 5 anos no modelo de sítio empregado no software SISPINUS desenvolvido pela EMBRAPA.

\section{Ajuste do Modelo de Clutter}

As estatísticas do ajuste do modelo de Clutter são apresentadas na Tabela 4.

Santana etal.(2005), em pesquisa com Eucalyptus grandis, encontraram valores de $R^{2}{ }_{a j}$ e Syx\% próximos ao do presente trabalho. Valores de $R^{2}{ }_{a j}$ maiores que 0,80 também têm sido observados em outras pesquisas com o modelo de Clutter (CRUZ et al., 2008; GORGENS et al., 2007; SOARES et al., 2004; SOARES; LEITE, 2000). $\mathrm{Na}$ Figura 3(a) e 3(b) são apresentadas as distribuições dos resíduos do modelo de volume e do modelo de área basal. Não se identificaram tendências no gráfico de resíduos.
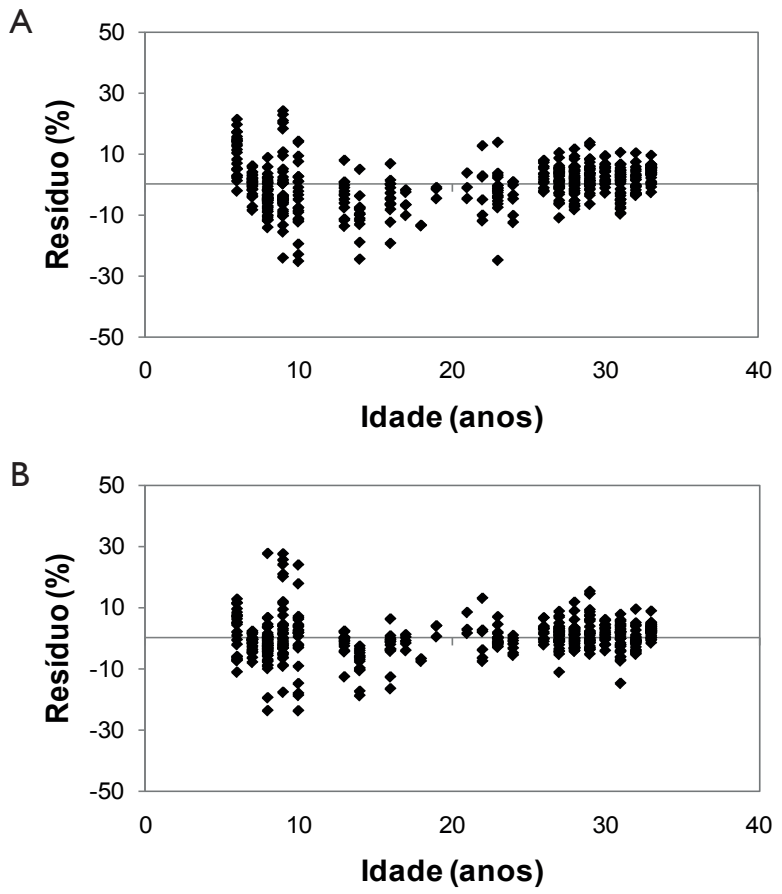

A- Modelo de volume; B- Modelo de área basal

FIGURA 3 Distribuição dos resíduos para os dois modelos de Clutter.

FIGURE 3 Residual distribution for the two Clutter models.

\section{Simulação do crescimento e produção sem considerar o desbaste}

As curvas de produção para o volume e área basal, considerando os sítios 20, 23 e 26, são apresentadas na Figura 4(a) e 4 (b).

TABELA 4 Coeficientes e estatísticas dos ajustes dos modelos de Clutter para projetar a produção em volume $\left(\mathrm{m}^{3} / \mathrm{ha}\right)$ e a área basal ( $\left.\mathrm{m}^{2} / \mathrm{ha}\right)$. TABLE 4 Coefficients and statistics for the Clutter model to predict volume $\left(\mathrm{m}^{3} / \mathrm{ha}\right)$ and basal area $\left(\mathrm{m}^{2} / \mathrm{ha}\right)$ production.

\begin{tabular}{cccc}
\hline Modelo & $\mathrm{R}_{\mathrm{aj}}^{2}$ & $\mathrm{~S}_{\mathrm{yx}}$ & $\mathrm{S}_{\mathrm{yx}}(\%)$ \\
\hline $\ln \left(V_{2}\right)=2,9475+\frac{-10,3349}{I_{2}}+0,0068 \cdot S+0,9727 \cdot \ln \left(A B_{2}\right)$ & 0,9948 & $27,46 \mathrm{~m}^{3} / \mathrm{ha}$ & 6,13 \\
$\ln \left(A B_{2}\right)=\ln \left(A B_{1}\right) \cdot\left(\frac{l_{1}}{I_{2}}\right)+4,6834 \cdot\left(1-\frac{l_{1}}{I_{2}}\right)+0,0055 \cdot\left(1-\frac{l_{1}}{l_{2}}\right) \cdot S$ & 0,9555 & $2,22 \mathrm{~m}^{2} / \mathrm{ha}$ & 5,58 \\
\hline
\end{tabular}



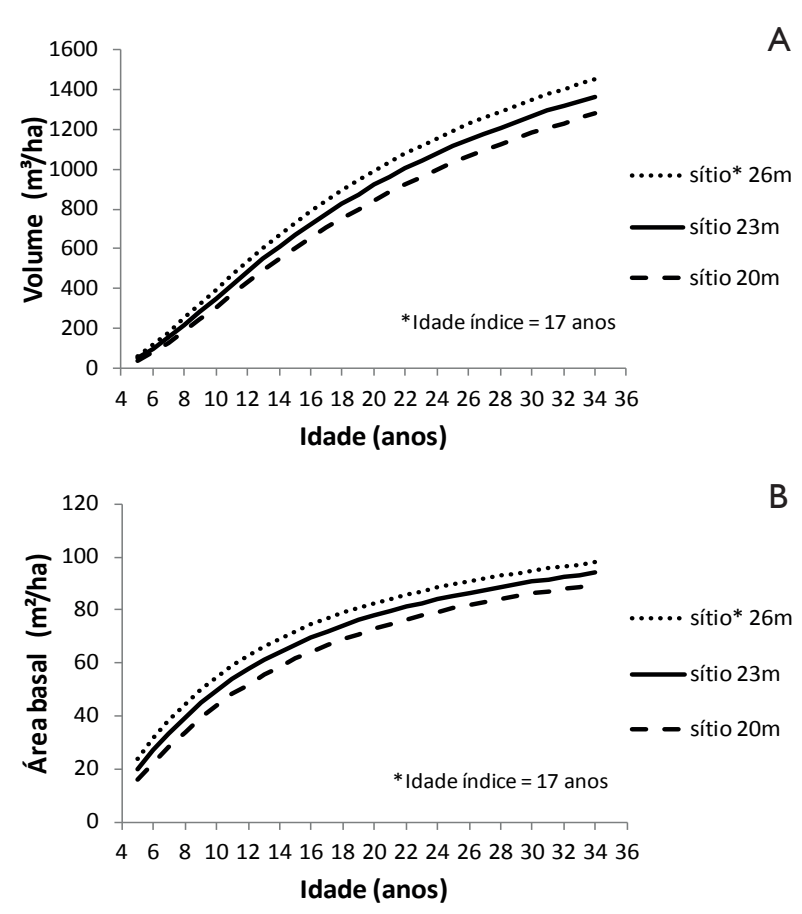

FIGURA 4 Curvas de produção para o volume e área basal para cada índice de sítio.

FIGURE 4 Volume and basal area production curves for each site index.

O IMA aos 17 anos, idade de corte utilizada pela empresa, foi de $4 \mathrm{I}, 54 \mathrm{~m}^{3} \cdot \mathrm{ha}^{-1} \cdot \mathrm{ano}^{-1} 45,7 \mathrm{I} \mathrm{m}^{3} \cdot \mathrm{ha}^{-1} \cdot \mathrm{ano}^{-1}$ e $49,7 \mathrm{l} \mathrm{m}^{3} \cdot \mathrm{ha}^{-1} \cdot$ ano $^{-1}$, para os sítios $20 \mathrm{~m}, 23 \mathrm{~m}$ e 26 metros, respectivamente. Esses valores estão dentro do intervalo do que foi observado por Mainardi et al. (1996) para o Pinus taeda. Para esses autores, o IMA, aos 16 anos, variou de $15,23 \mathrm{~m}^{3} \cdot \mathrm{ha}^{-1} \cdot \mathrm{ano}^{-1}$ no pior índice de sítio a $56,94 \mathrm{~m}^{3} \cdot \mathrm{ha}^{-1} \cdot$ ano $^{-1}$ para o melhor.

Na Figura 5(a) e 5(b), são apresentadas as curvas de IMA e ICA para o volume e área basal, respectivamente.

$\mathrm{Na}$ Figura 5, os pontos onde as curvas de ICA e IMA se cruzam, são indicadas com linhas verticais. Esses pontos para o volume são 19,20 e 21 anos, para os sítios 20,23 e 26 metros, respectivamente, confirmando o fundamento biológico de que quanto melhor o sítio, menor a idade técnica de corte (ITC). As ITC para a área basal são 9, 10 e II anos, as quais indicam a idade ideal para o desbaste, próximas da idade de desbaste de 10 anos, empregada pela empresa. Nos povoamentos florestais, as árvores começam a competir, primeiramente, em relação à área basal do que em volume, justificando a realização do desbaste antes do povoamento atingir a ITC no volume.
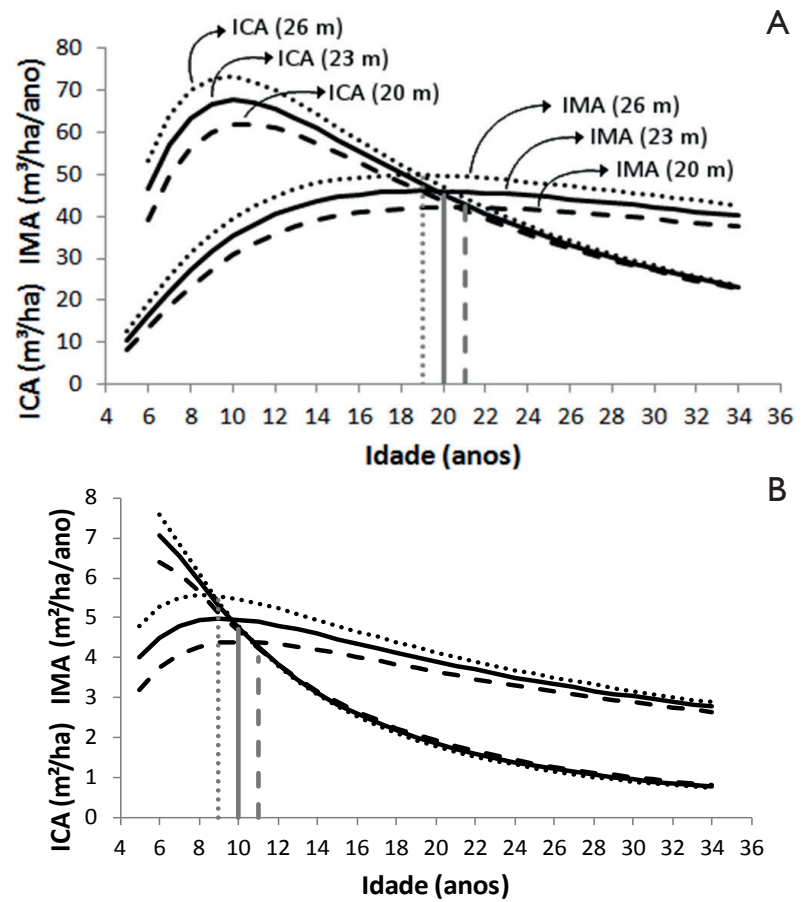

FIGURA 5 Curvas de incrementos anuais para o volume e área basal nos índices de sítio 20, 23 e 26 m.

FIGURE 5 Annual increments curves for volume and basal area on site index 20,23 and $26 \mathrm{~m}$.

\section{Simulação do crescimento e produção considerando o desbaste}

Considerando o desbaste de $40 \%$ para a área basal, aos nove anos de idade, a ITC para o volume passa a ser 23, 24 e 25 anos, respectivamente para cada índice de sítio. Na Figura 6, são apresentadas as curvas construídas com base nas equações ajustadas.

\section{Comparação do volume projetado em relação ao volume real}

$\mathrm{Na}$ Figura 7, são apresentadas projeções do modelo ajustado em comparação com remedições de alguns talhões com diferentes idades. Observa-se que $\circ$ modelo fornece estimativas do volume, na maioria das vezes, próximas aos valores reais. Na Figura 7(a), um talhão de idade avançada, que sofreu dois desbastes, o modelo apresenta um erro de $22,1 \mathrm{~m}^{3} \cdot \mathrm{ha}^{-1}(3,2 \%)$, na idade de 29 anos, em relação ao que foi medido no inventário. Nesse caso, a simulação foi realizada a partir da área basal na idade de 5 anos. Melhores estimativas podem ser obtidas, usando dados de idades mais próximas da idade de projeção. 


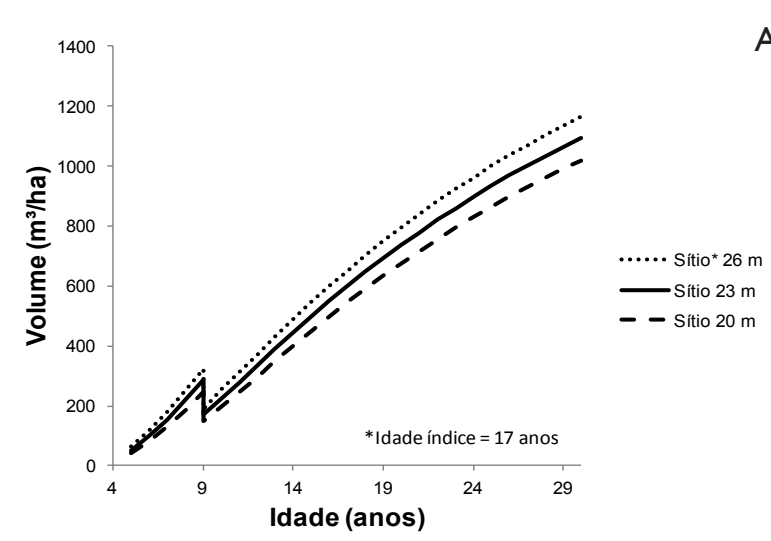

A

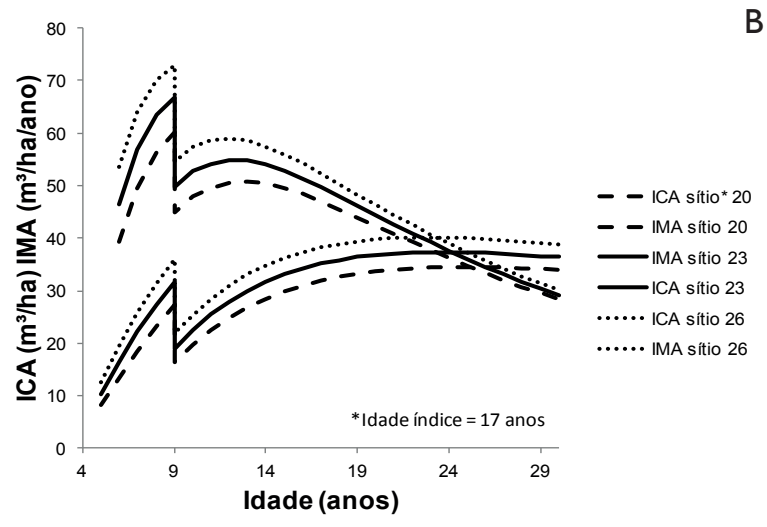

FIGURA 6 Curvas de produção e de incrementos anuais para o desbaste aos 9 anos.

FIGURE 6 Production and annual increments curves for thinning at 9 years.

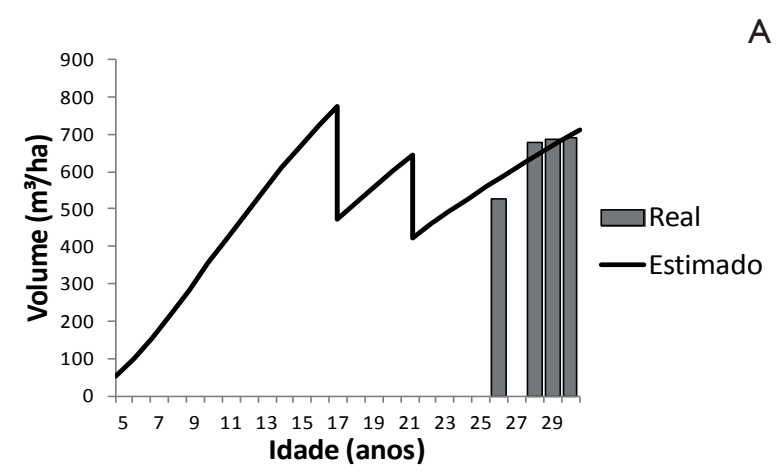

A
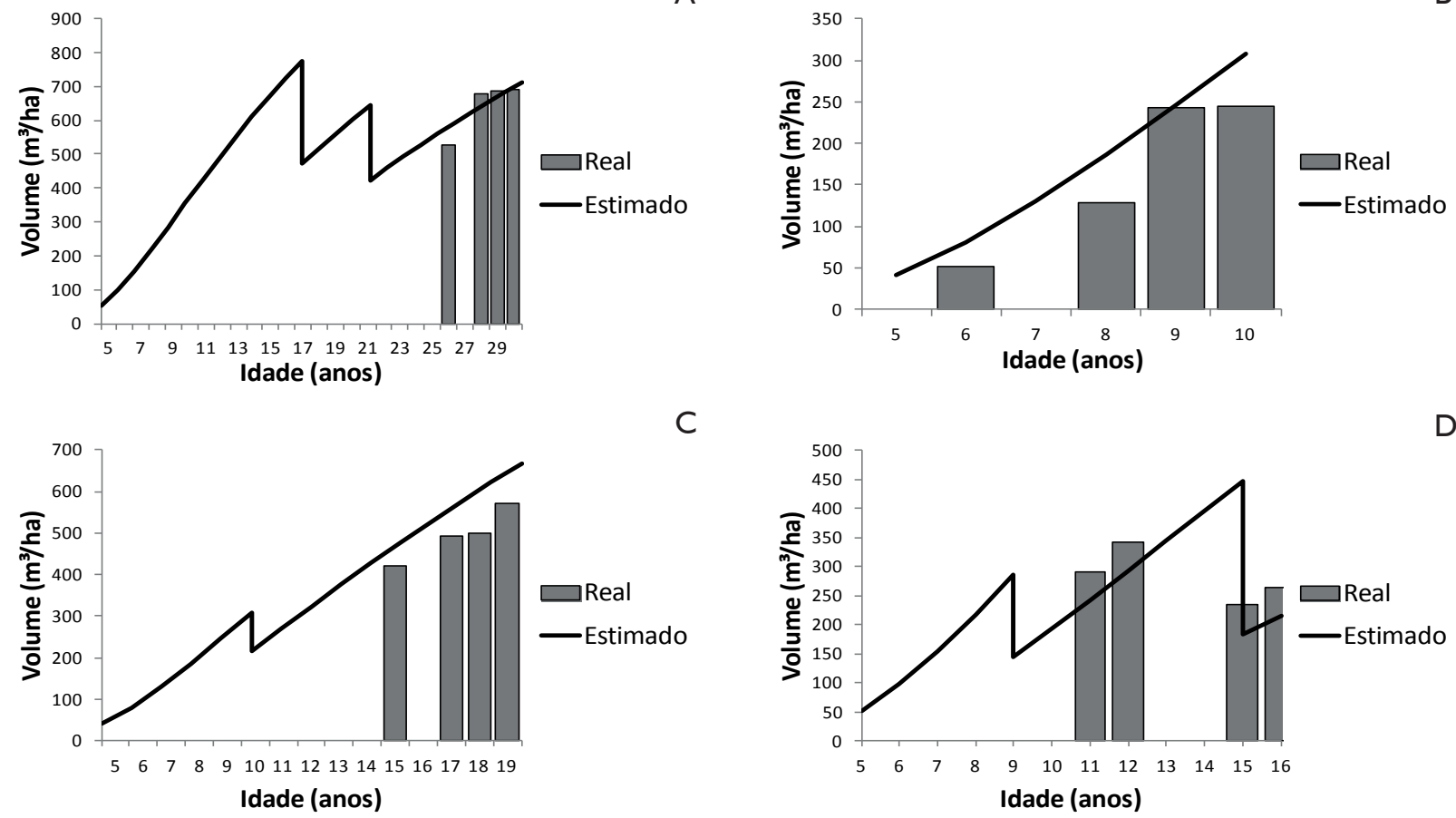

FIGURA 7 Exemplo de projeções do volume estimado em relação ao volume real.

FIGURE 7 Example of volume estimate projections in relation to the observed volume.

\section{CONCLUSÕES}

Os modelos de Silva-Bailey, Chapman-Richards, Weibull, Mitscherlich, Clutter-Jones e Schumacher, testados para expressar o índice de sítio em povoamentos de Pinus taeda, para o local estudado, são considerados adequados, sendo selecionado o de Silva-Bailey por ter apresentado erro menor em relação aos demais.

O modelo de Clutter ajusta-se bem à base de dados, sendo considerado eficiente para estimar o crescimento e a produção $\mathrm{em} \mathrm{m}^{3} \cdot \mathrm{ha}^{-1}$, do ponto de vista estatístico e biológico;

A ITC (idade técnica de corte) para a área basal ocorre antes da ITC para o volume como esperado. Isso justifica a realização de desbastes antes dos povoamentos atingirem o máximo incremento médio anual em volume.

O modelo de Clutter ajustado pode ser empregado para estimar o volume, com ou sem desbastes, em $\mathrm{m}^{3}$.ha- $\mathrm{a}^{-1}$ para povoamentos de Pinus taeda, na região norte do estado de Santa Catarina. 


\section{REFERÊNCIAS}

CAMPOS, J. C. C.; LEITE, H. G. Mensuração florestal: perguntas e respostas. Viçosa, MG: UFV, 2009.

CLUTTER, J. C.; FORTSON, J. C.; PLENAAR, L. V.; BRISTER, G. H.; BAILEY, R. L. Timber management: a quantitative approach. $3^{\text {rd }}$ ed. New York: J. Willey, 1983. 333 p.

CLUTTER, J. L. Compatible growth and yields models for loblolly pine. Forest Science, Champaign, v. 9, n. 3, p. 344-37I, 1963.

CRUZ, J. P.; LEITE, H. G.; SOARES, C. P. B.; CAMPOS, J. C. C.; SMIT, L.; NOGUEIRA, G. S.; OLIVEIRA, M. L. R. Modelos de crescimento e produção para plantios comerciais jovens de Tectona grandis em Tangará da Serra, Mato Grosso. Revista Árvore, Viçosa, v. 32, n. 5, p. 821 828, 2008.

DIAS, A. N. Modelagem e avaliação econômica de plantações de eucalipto submetidas a desbaste. 2000. 82 p. Dissertação (Mestrado em Ciência Florestal) Universidade Federal de Viçosa, Viçosa, 2000.

DIAS, A. N. Um modelo para gerenciamento de plantações de eucalipto submetidas a desbaste. 2005. I47 p. Tese (Doutorado em Ciência Florestal) Universidade Federal de Viçosa, Viçosa, 2005.

DIAS, A. N.; LEITE, H. G.; CAMPOS, J. C. C.; COUTO, L.; CARVALHO, A. F. Emprego de um modelo de crescimento e produção em povoamentos desbastados de eucalipto. Revista Árvore, Viçosa, v. 29, n. 5, p. 731-739, 2005a.

DIAS, A. N.; LEITE, H. G.; SILVA, M. L.; CARVALHO, A. F. Avaliação financeira de plantações de eucalipto submetidas a desbaste. Revista Árvore, Viçosa, v. 29, n. 3, p. 419429, 2005b.

GORGENS, E. B.; LEITE, H. G.; NOGUEIRA, G. S.; DIAS, A. N. Tendência de crescimento de povoamento de eucalipto após aplicação de desbaste. Revista Árvore, Viçosa, v. 3I, n. 5, p. 879-885, 2007.

MAINARDI, G. L.; SCHNEIDER, P. R.; FINGER, C. A. G. Produção de Pinus taeda L. na região de Cambará do Sul, RS. Ciência Florestal, Santa Maria, v. 6, n. I, p. 39-52, 1996.
MARTINS, E. F. P.; SILVA, J. A. A.; FERREIRA, R. L. C.; JANKOVSKI, T.; BRITO, C. C. R. Curvas de índice de sítio para leucena [Leucaena leucocephala (Lam.) de Wit.] no agreste de Pernambuco. Ciência Florestal, Santa Maria, v. I7, n. 4, p. 365-376, 2007.

RESENDE, R. R.; VALE, A. B.; SOARES, T. S.; SILVA, M. L.; COUTO, L.; VALE, R. S. Emprego de um modelo de crescimento e produção para determinação da rotação em povoamentos de eucalipto. Revista Árvore, Viçosa, v. 28, n. 2, p. $219-225,2004$.

SANTANA, C.; MELLO, A. A.; EISFELD, R. L.; SANQUETTA, C. R. sistema de equações para simulação do crescimento e da produção em povoamentos de Eucalyptus grandis Hill ex Maiden sem desbaste baseado no modelo de clutter. Ambiência, Guarapuava, v. I, n. 2, p. 239-256, 2005.

SCOLFORO, J. R. S.; MACHADO, S. A. Curvas de índice de sítio para plantações de Pinus elliottii nos Estados do Paraná e Santa Catarina. Revista Floresta, Curitiba, v. 18, n. 12, p. 140-158, 1988.

SELLE, G. L.; SCHNEIDER, P. R.; FINGER, C. A. G. Classificação de sítio para Pinus taeda L., através da altura dominante, para a região de Cambará do Sul, RS, Brasil. Ciência Florestal, Santa Maria, v. 4, n. I, p. 77-95, 1994.

SOARES, C. P. B.; LEITE, H. G. Predição da produção de madeira de eucalipto em região com alta variabilidade pluviométrica. Scientia Forestalis, Santa Maria, n. 58, p. 4I-48, 2000.

SOARES, C. P.B.; LEITE, H. G.; OLIVEIRA, M. L. R.; CARVALHO, A. Especificação de um modelo de crescimento e produção. Revista Árvore, Viçosa, v. 28, n. 6, p. 83I-837, 2004.

TÉO, S. J.; BRESSAN, D. R.; COSTA, R. H. Uso de modelos estatísticos para classificação de sítios em povoamentos de Pinus taeda na região de Caçador, SC. Floresta, Curitiba, v. 4 I, n. I, p. I79-I88, 201 I.

TONINI, H.; FINGER, C. A. G.; SCHNEIDER, P. R.; SPATHELF, $P$. Índice de sítio para Pinus elliottii Engelm, em três unidades de mapeamento de solo, nas regiões da serra do sudeste e litoral, no Estado do Rio Grande do Sul, Brasil. Ciência Florestal, Santa Maria, v. I2, n. 2, p. 6I-73, 2002.

VANCLAY, J. K. Modelling forest growth and yield: applications to mixed tropical forests. Wallingford: $C A B$ International, 1994. 312 p. 